\title{
Stationary and Recurrent Properties of Atrial Fibrillation Conduction Patterns in Goat
}

\author{
Arne van Hunnik ${ }^{1}$, Stef Zeemering ${ }^{1}$, Piotr Podziemski ${ }^{1}$, Jorik Simons ${ }^{1}$, Giulia Gatta ${ }^{1}$, Bart Maesen ${ }^{1}$, \\ Marion Kuiper $^{1}$, Sander Verheule ${ }^{1}$, Ulrich Schotten ${ }^{1}$ \\ ${ }^{1}$ Maastricht University, Maastricht, The Netherlands
}

\begin{abstract}
Introduction. Electrical mapping of the atria is used to assess the substrate of atrial fibrillation (AF). Targeted ablation of the AF substrate assumes spatiotemporal stationarity. In this study we analyzed long AF recordings of AF using high-density contact mapping.

Methods. In 12 goats with stable AF 10 successive $60 \mathrm{~s}$ files were recorded, within a single AF episode. AF cycle length, fractionation index (FI), lateral dissociation, conduction velocity, breakthroughs and preferentiality of conduction (Pref) were assessed to construct AF-property maps. The Pearson correlation coefficient (PCC) between AF-property maps of consecutive recordings was calculated. Recurrence plots and recurrence quantification analysis were used to identify recurrent patterns.

Results Spatiotemporal stationarity for the 6 properties were high, PCC ranged from $0.66 \pm 0.11$ for Pref to $0.98 \pm 0.01$ for FI. The PCC is not affected by the time delay between files. Yet, highly dynamic patterns were found. Recurrence plots revealed few (1.6 \pm 0.7$)$ recurrent patterns in individual animals.

Conclusions AF properties were stationary in stable $A F$. This cannot be attributed to stable recurrent conduction patterns. during This suggests that spatial properties of the atrium determine AF properties.
\end{abstract}

\section{Introduction}

Mapping of conduction patterns has been of fundamental importance to understand mechanisms that maintain cardiac arrhythmias. The importance of cardiac mapping in clinical practice was demonstrated by Haïssaguerre's et al. [1] pivotal finding that atrial fibrillation (AF) was often initiated from the pulmonary veins (PV) and that ablation of ectopic sites in the PVs terminated AF. However, many patients experience recurrences of $\mathrm{AF}$ in the months following a PV isolation [2], suggesting that other regions in the atria may contribute to $\mathrm{AF}$ perpetuation as well.

Detailed mapping studies of AF, in both animal models [3] and humans [4], have described fast, irregular and seemingly random conduction patterns. Several electrophysiological parameters have been used to detect local sources of AF. [5] Complex fractionated atrial electrograms (CFAE) and high frequency zones are thought to reflect driver sites of rotational or focal activity or to correlate with areas demonstrating high complexity of AF. Unlike such electrogram parameters, with focal impulse and rotor mapping (FIRM) conduction patterns are identified that putatively describe focal and rotational activity. [6] Unfortunately, targeting these substrate parameters had variable outcomes in clinical trials and need further validation.[7-9]

A potential factor contributing to the limited success rates are the limitations of the mapping techniques used. The atrial surface can only be mapped with a limited time and spatial resolution. A limited spatial resolution may lead to misinterpretation of conduction patterns.[10] Relatively short recordings are acquired when higher spatial resolution is obtained by point-by-point mapping. It is unknown whether these recordings are representative for longer episodes of AF. Also, it is largely unexplored whether AF driver sites are stable across different $\mathrm{AF}$ episodes.

In this study we analysed left atrial epicardial highdensity recording in goats with stable AF. We studied the degree of stationarity of local AF properties. Moreover, we analysed complexity and stability of conduction patterns, and rotational activity.

\section{Methods}

In total 12 goats weighing $60 \pm 9.8 \mathrm{~kg}$ were included. AF was induced by repetitive burst pacing $(1 \mathrm{~s}, 50 \mathrm{~Hz}, 2$ times threshold with a maximum of $10 \mathrm{~V}$ ). AF was subsequently maintained for 3-4 weeks. In an open chest experiment, unipolar electrograms were recorded $(1.039 \mathrm{kHz}$ sampling rate, bandwidth of $0.1-408 \mathrm{~Hz}$ and $\mathrm{AD}$ resolution of 16bit) with 249 electrodes $(2.4 \mathrm{~mm}$ inter-electrode distance, $14.3 \mathrm{~cm}^{2}$ surface area) on the left atrium. AF was recorded for 10 minutes in ten 60-second files AF. A custom-made tool was used analyse the signals (MATLAB). Local deflections, activation times, and unipolar fractionation index (FI) were identified using a probabilistic annotation algorithm, as previously described. [11,12] Based on the activation times, the AF cycle length (AFCL) and conduction direction and velocity (CV) were determined. 
The preferential direction of conduction (Pref) was calculated for each electrode (Pref=1-(circular variance of all conduction vectors)). Epicardial lateral dissociation (LD) was calculated as the maximal time difference of activation times between neighbouring electrodes. AF property maps 5 different parameters, i.e. FI, AFCL, LD, $\mathrm{CV}$, and Pref were obtained for each individual animal. Per animal and parameter, the spatial Pearson correlation coefficient (PCC) between all property maps were determined. We analysed waves during AF as previously described by Zeemering et al. Conduction paths within waves were determined as the shortest contiguous trajectory between a starting and end point. [11] Trajectory with $\geq 1$ intersection(s) were considered to be re-entrant circuits (RC).

Next, we analysed the recurrent conduction patterns in 60 -second-recordings. We considered a pattern to be recurrent if the wave front(s);1) reached the same point in space, 2) propagated in the same direction, and 3) had a similar shape. To identify recurrent events, we used the linear phase between activation times. Note: Hilbert transformation or time embedding of the raw electrogram are not needed as local activation times are fundamental base of this approach. The difference in phase at the electrode level was computed between every sample. The average phase difference (aPD) was computed based on all 249 phase differences. Recurrent patterns were identified at an aPD of $<\pi / 4$, corresponding to $1 / 8$ of the AFCL. The rate of recurrent patterns was calculated as the ratio of the number of observed recurrences to the number of expected recurrences if the pattern was fully recurrent.

Data are presented as mean \pm sd. Data were tested for normality using a Kolmogorov-Smirnov test. Intra- and inter-episode correlation between property maps was assessed using Pearson correlation coefficient (PCC). A Bonferroni's correction was applied to correct for multiple comparisons. Inter-episode correlation differences were tested with a Wilcoxon rank sum test.

\section{Results}
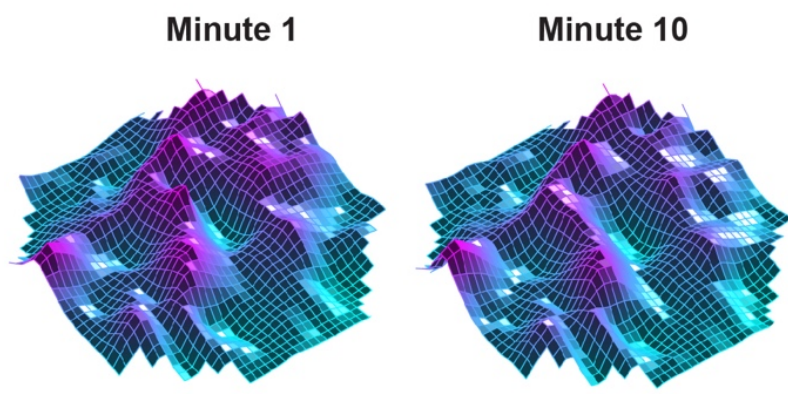

Figure 1. Unipolar fractionation index property map from the first and the tent minute of a 10 -minute AF recording. The purple peaks reflect electrodes with high levels of FI, green zones reflect low levels of FI.
In figure 1 two property maps for the fractionation index is presented. FI was the parameter with highest level of stationarity (figure 1) but all property maps presented very reproducible spatial distributions. The average PCCs was very high and ranged from $0.66 \pm 0.11$ for Pref up to $0.98 \pm 0.01$ for FI, (table 1). To investigate if the high PCC were due to chance we broke the spatial coherence of the maps by randomly reassigning the electrodes within the map. This test diminished the average PCCs to almost 0.

Table 1. Print sizes for different parts of the manuscript.

\begin{tabular}{lcc}
\hline Text & $\begin{array}{c}\text { PCC } \\
(\mathrm{r})\end{array}$ & $\begin{array}{c}\text { PCC } \\
\mathrm{p} \text {-value }\end{array}$ \\
\hline FI & $0.983 \pm 0.01$ & $\mathrm{p}<0.001$ \\
AFCL $(\mathrm{ms})$ & $0.956 \pm 0.04$ & $\mathrm{p}<0.001$ \\
LD $(\mathrm{ms})$ & $0.968 \pm 0.01$ & $\mathrm{p}<0.001$ \\
CV $(\mathrm{cm} / \mathrm{s})$ & $0.926 \pm 0.06$ & $\mathrm{p}<0.001$ \\
Pref & $0.655 \pm 0.11$ & $\mathrm{p}<0.001$ \\
\hline
\end{tabular}

We also investigated if distance in time affected the PCC between property maps but no changes in PCC occurred for all parameters. Next we investigated if the spatiotemporal stationarity would be affected between AF episodes. Two episodes were closely apart ( 2 minutes) and a third episode was recoded after $\sim 1$ hour of AF interruption. A short AF interruption had no substantial impact on PCC but larger changes occurred after the 60minute interruption. The decrease of PCC was significant for AFCL, CV, and FI.
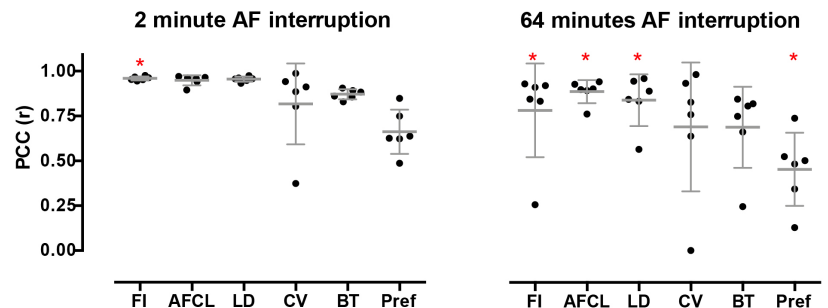

Figure 2. Inter-episode correlation. Red asterisks mean a significant lower PCC compared to intra-episode correlation.

The stationary property maps could have been dependent on a beat-to-beat level or at somewhat longer timespans. We therefore examined AF dynamics. Beat-tobeat AF dynamics resulted in an AFCL change of $20 \pm 3.7 \mathrm{~ms}$ and conduction direction varied with $66 \pm 10$ degrees. $7.7 \pm 2.3$ waves were present per cycle. 453 reentrant trajectories were found across all files with only 25 lasting longer than 2 rotations. On average re-entrant activity was present for $9.2 \pm 11 \%$ with a lifespan of $149 \pm 18 \mathrm{~ms}$. These finding demonstrated a large beat-to- 
beat variability with high dynamic patterns that cannot explain stationary AF properties.

However, frequent recurrent patterns that dominate the property maps could have dictated stationarity. We therefore investigated the occurrence of recurrent $\mathrm{AF}$ patterns with the use of recurrence plots. An example is presented in figure 2.9 out of 12 goats no frequent or long-

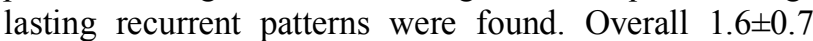
recurrent patterns per goat were found, with either peripheral or BT origins. $64 \%$ of the reentrant circuits with $>2$ rotations were identified as recurrent events. No predominant origin of conduction pattern was found.

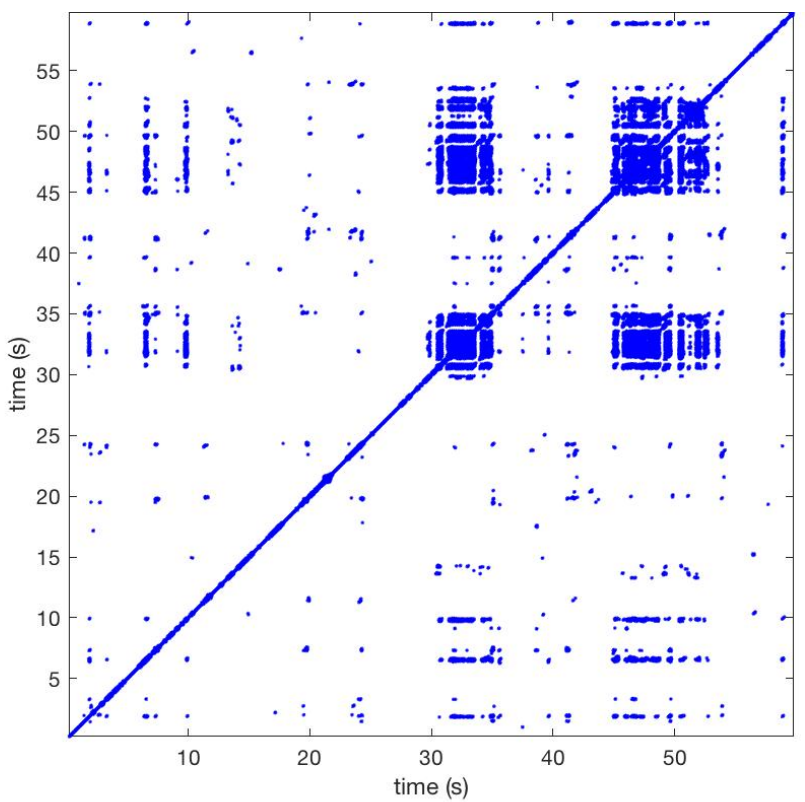

Figure 2. Recurrent pattern analysis of a 60 second AF files in recorded in a goat with 3-4 weeks of AF. Most of the time no recurrent patterns were present. 3 blocks can be acknowledged indicating a single recurrent pattern (at 32s, $48 \mathrm{~s}$, and $52 \mathrm{~s}$ ). The recurrence block identified a repetitive breakthrough pattern.

\section{Discussion}

In the goat model of persistent $\mathrm{AF}$, we demonstrated that in 60-second-recordings the spatiotemporal distributions of AF parameters are stationary if measured within a single $\mathrm{AF}$ episode. A short interruption of an $\mathrm{AF}$ episode had little effect on this stationary behaviour but interruption of longer duration changed AF parameters significantly. 60-second recurrence analysis illustrated that recurrent patterns were scarce and only found in a minority of the animals. AF was also shown to have highly dynamic behaviour at a beat-to-beat level. Therefore, stationary spatiotemporal properties could not be attributed to stable recurrent conduction patterns during $\mathrm{AF}$ suggesting that the atrial myocardium may determine average $\mathrm{AF}$ properties even when the conduction patterns are highly variable.

Complex fractionated electrograms (CFAE) were reported to occur during induced $\mathrm{AF}$ at sites of conduction block in Wolf-Parkinson-White patients and therefore reflect areas with higher complexity of AF.[13] Consequently, they might reflect zones that act as sources for AF. It was hypothesized that CFAE sites might be suitable targets for AF ablation.[14] Initially high success rates were reported but recent randomized controlled trials failed to show superiority of PV isolation + CFAE ablation above PV isolation alone.[2] In other studies, high dominant frequency (DF) was found to associate with rotor sites in cholinergic AF in sheep hearts.[15] This notion led to hypothesis that ablating DF sites might be a valid target for AF ablation.[15] Indeed, the presence of high frequency sites inversely correlated to procedural success rates [8] but targeting DF sites did not consistently lead to improvement of clinical outcome.[8] It is unclear whether these negative outcomes entail that CFAE and high DF indicate bystander sites instead of $\mathrm{AF}$ driver sites or are due to technical limitations of the methods applied. Irrespective of the applied methods, the intrinsic assumption entail that driver sites are stable in time and space. The question is whether the recording lengths are sufficient to capture potential transient appearances of CFAE's or DF's. Continuous recordings of bipolar contact electrograms showed that about $75 \%$ of CFAE's remain stable in time.[12] Similarly, we found very high spatiotemporal stationarity for both FI and AFCL. Recordings of 60 seconds within a single episode of AF were more than sufficient to reliably capture patterns.

Both AFCL and FI are parameters that are derived from single electrograms and reflect properties of a limited area. Parameters that account for spatial characteristics of conduction, had high stationary properties too. Stationarities were lower when AF patterns were taken into account. Preferentiality of conduction direction was subject to largest variability, indicative of dynamic conduction patterns.

In general, $\mathrm{AF}$ wave and recurrence analysis revealed multiple propagating wave fronts with a small number of recurrent patterns. Hence, it is unlikely that the high degree of stationary of AF property maps is caused by stable and frequent conduction patterns. Therefore, we conclude that the atrial structure and local electrophysiological properties are likely to determine average AF properties even if the conduction patterns as such are very variable. This might imply that the relatively large variation in $\mathrm{AF}$ complexity (3.8-11 waves/cycle), among animals without substantial structural remodeling [3], may - at least partly - be driven by the individual atrial architecture.

If AF properties would only be determined by the atrial architecture, then the initiation conditions should not affect AF properties. We therefore examined the effect of different episodes of AF. We found that stationarity is conserved if two AF episodes are relative close in time ( 
2 minutes). This implies that atrial structure has a prominent role on conduction properties during AF. However, stationarity of AF properties was lower when AF was interrupted $>1$ hour. Because atrial structure does not change in this time interval, we propose that some form of acute recovery from electrical remodeling affected the atrial substrate. This observation might be of clinical importance since some symptomatic AF patients are cardioverted to sinus rhythm before AF ablation. Reinduction of AF during the procedure may reveal other AF patterns or drivers than originally were present with the risk that the leading mechanism cannot be identified.

Recurrence quantification analysis is a technique to identify recurrent states in non-linear dynamical systems. In AF, recurrence analysis has been used mostly for (fractionated) electrogram morphology.[16] Here, we introduce a method accounting for both directionality and complexity of conduction patterns. An important finding is that recurrent patterns were scarce in the overall data set. Recurrent patterns mainly occurred in animals with a low degree of AF complexity. The introduced recurrence analysis allows addressing AF on a large time scale and may generate new ideas about AF perpetuation. For instance, the identification of a recurrent period within an episode of AF could indicate maintenance of AF by large circuits, while non-recurrent periods reflect more localized mechanisms. Alternatively, recurrent periods could reflect large transient reentrant circuits that act as oscillators of the fibrillation process. These resonators may then speed up fibrillation rate with consequent breakdown in distant areas of the atria. Otherwise, recurrent patterns may also occur before cardioversion of AF when AF complexity becomes low.

An obvious limitation of this study is that the recordings were performed in the goat model of persistent AF in the presence of limited structural remodeling. Additional investigations are required to investigate whether similar patterns occur in patients with AF. Also, the effect of the degree of structural remodeling on recurrent patterns should be addressed in future studies.

\section{References}

1 Haissaguerre M, Jais P, Shah DC, et al. Spontaneous initiation of atrial fibrillation by ectopic beats originating in the pulmonary veins. $N$ Engl J Med 1998;339:659-66. doi:10.1056/NEJM199809033391003

2 Verma A, Jiang C-Y, Betts TR, et al. Approaches to catheter ablation for persistent atrial fibrillation. $N$ Engl J Med 2015;372:1812-22. doi:10.1056/NEJMoa1408288

3 Verheule S, Tuyls E, van Hunnik A, et al. Fibrillatory conduction in the atrial free walls of goats in persistent and permanent atrial fibrillation. Circ Arrhythm Electrophysiol 2010;3:590-9. doi:10.1161/CIRCEP.109.931634

4 Allessie MA, de Groot NMS, Houben RPM, et al. Electropathological substrate of long-standing persistent atrial fibrillation in patients with structural heart disease: longitudinal dissociation. Circ Arrhythm Electrophysiol 2010;3:606-15. doi:10.1161/CIRCEP.109.910125

5 Nedios S, Sommer P, Bollmann A, et al. Advanced mapping systems to guide atrial fibrillation ablation: electrical information that matters. J Atr Fibrillation 2016;8:1337. doi:10.4022/jafib. 1337

6 Narayan SM, Krummen DE, Shivkumar K, et al. Treatment of atrial fibrillation by the ablation of localized sources: CONFIRM (Conventional Ablation for Atrial Fibrillation With or Without Focal Impulse and Rotor Modulation) trial. J Am Coll Cardiol 2012;60:628-36. doi:10.1016/j.jacc.2012.05.022

7 van der Does LJME, de Groot NMS. Inhomogeneity and complexity in defining fractionated electrograms. Heart Rhythm 2017;14:616-24. doi:10.1016/j.hrthm.2017.01.021

8 Gadenz L, Hashemi J, Shariat MH, et al. Clinical role of dominant frequency measurements in atrial fibrillation ablation - A Systematic Review. J Atr Fibrillation 2017;9:1548. doi:10.4022/jafib. 1548

9 Parameswaran R, Voskoboinik A, Gorelik A, et al. Clinical impact of rotor ablation in atrial fibrillation: a systematic review. Europace 2018;20:1099-106.

doi:10.1093/europace/eux370

10 Kuklik P, Zeemering S, van Hunnik A, et al. Identification of rotors during human atrial fibrillation using contact mapping and phase singularity detection: technical considerations. IEEE Trans Biomed Eng 2017;64:310-8. doi:10.1109/TBME.2016.2554660

11 Zeemering S, Maesen B, Nijs J, et al. Automated quantification of atrial fibrillation complexity by probabilistic electrogram analysis and fibrillation wave reconstruction. Conf Proc IEEE Eng Med Biol Soc 2012;2012:6357-60. doi:10.1109/EMBC.2012.6347448

12 Lau DH, Maesen B, Zeemering S, et al. Indices of bipolar complex fractionated atrial electrograms correlate poorly with each other and atrial fibrillation substrate complexity. Heart Rhythm 2015;12:1415-23. doi:10.1016/j.hrthm.2015.03.017

13 Konings KTS, Smeets JLRM, Penn OC, et al. Configuration of unipolar atrial electrograms during electrically induced atrial fibrillation in humans. Circulation 1997;95:1231-41. doi:10.1161/01.CIR.95.5.1231

14 Nademanee K, McKenzie J, Kosar E, et al. A new approach for catheter ablation of atrial fibrillation: mapping of the electrophysiologic substrate. J Am Coll Cardiol 2004;43:2044-53. doi:10.1016/j.jacc.2003.12.054

15 Mandapati R, Skanes A, Chen J, et al. Stable microreentrant sources as a mechanism of atrial fibrillation in the isolated sheep heart. Circulation 2000;101:194-9. doi:10.1161/01.CIR.101.2.194

16 NG J, Gordon D, Passman RS, et al. Electrogram morphology recurrence patterns during atrial fibrillation. Heart Rhythm 2014;11:2027-34. doi:10.1016/j.hrthm.2014.08.002

Address for correspondence. Arne van Hunnik. P.O. Box 616 6200 MD Maastricht The Netherlands a.vanhunnik@maastrichtuniversity.nl 